\title{
Positrons from Gamma-Rays
}

A report of the direct observation of positrons ejected from lead by hard gamma-rays has been published. ${ }^{1} \mathrm{~A}$ source of radiothorium with its disintegration products was used. To obtain a well-defined beam the gamma-rays were collimated by allowing them to pass through a half-inch hole in a series of lead blocks totaling 18 inches in length. That the beam actually was well defined is shown by the fact that most of the observed tracks appeared to originate within a rather narrow region, about $3 \mathrm{~cm}$ wide. In this series of photographs the beam passed vertically downward through the side wall of a Wilson chamber across which were placed a $2 \mathrm{~mm}$ lead sheet, and below this a $0.5 \mathrm{~mm}$ sheet of aluminum. A magnetic field of 430 gauss normal to the face of the chamber and uniform to within 10 percent was used.

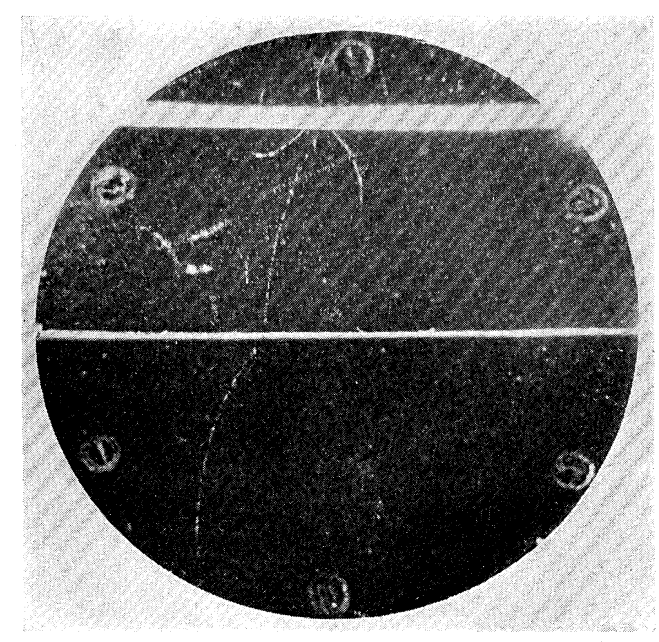

FIG. 1. A positron ejected from a lead plate by gammarays and passing through a $0.5 \mathrm{~mm}$ aluminum plate, showing a loss in energy. On the assumption that its mass is the same as that of a free electron the energy before penetrating the aluminum plate is 820,000 volts and afterward 520,000 volts.

A statistical study of the energy distribution of the positives and negatives produced by the gamma-radiation will throw considerable light on the nature of the processes involved in their production since the quantum energies of the photons are in this case well known. One of the most striking phenomena which have been observed in this experiment is the occasional simultaneous appearance of paired tracks consisting of one positive particle and one negative with a common point of origin. As a preliminary part of this study we have to date measured the energies of 22 such pairs ejected by the gamma-radiation, which has a strong band at $2.6 \times 10^{6}$ volts. Of the 22 pairs, 13 have kinetic energies (if the positron mass is assumed equal to that of the electron) from $10^{6}$ volts to $1.6 \times 10^{6}$; and only one pair has an energy greater than this, namely $1.8 \times 10^{6}$. Since isolated cases cannot be regarded with absolute certainty as associated pairs we may state the above result as statistical evidence suggesting a cut-off in the neighborhood of $1.6 \times 10^{6}$ volts.

The process which gives rise to the positrons is at present not known, but so far as these data go they are in accord with the view expressed by Blackett and Occhialini ${ }^{2}$ that the two particles may be formed by a process in which the energy of approximately one million volts required for the formation of a positron and a negative electron is supplied by the impinging radiation. Further, the energies of 13 single positively curved particles with which there is no trace of an associated negative have been measured. If we suppose that in each of these cases a pair was created but the positron happened to get most of the kinetic energy, then the maximum kinetic energy of these single particles should have the same upper limit as that of the pairs. Of the 13 there were 8 with energies from $10^{6}$ to $1.5 \times 10^{6}$, and none higher than the latter value. If, however, further work should reveal the presence of positrons whose energy differs from that of the incident photons by less than a million volts the above interpretation must be abandoned.

Skobelzyn ${ }^{3}$ in a detailed investigation of the space and energy distribution of the electrons ejected by hard gammarays found results not completely in agreement with the Klein-Nishina formula. Gray and Tarrant ${ }^{4}$ in studying the scattering of hard gamma-rays report the presence of a fluorescence radiation whose quantum energy apparently is confined to two bands in the neighborhood of $0.5 \times 10^{6}$ volts and $0.9 \times 10^{6}$ volts. Blackett and Occhialini have suggested that the origin of this fluorescence radiation may be the simultaneous annihilation of an electron and a positron. The effects observed in these two experiments increased with the hardness of the gamma-rays used; and they might be ascribed to the formation of positrons and electrons rather than to any inherent incorrectness in the KleinNishina formula, which considers scattering only from free negative electrons.

Since hard gamma-rays do produce positrons, the positrons observed by Chadwick, Blackett and Occhialini, ${ }^{5}$ and by Meitner and Philipp, ${ }^{6}$ who used a beam of neutrons with accompanying hard gamma-rays may be due only to the accompanying gamma-rays and not the neutrons. In fact Curie and Joliot ${ }^{7}$ by studying the yield of positrons as a function of the amount of filtering of the combined neutron and gamma-ray beam have arrived at just this conclusion.

Carl D. Anderson

Seth H. Neddermeyer

California Institute of Technology,

Pasadena, California, May 18, 1933.

${ }^{1}$ Anderson, Science 77, 432 (1933).

2 Blackett and Occhialini, Proc. Roy. Soc. A139, 699 (1933).

${ }^{3}$ Skobelzyn, Comptes Rendus 194, 1914 (1932).

${ }^{4}$ Gray and Tarrant, Proc. Roy. Soc. A136, 662 (1932).

${ }^{5}$ Chadwick, Blackett and Occhialini, Nature 131, 473 (1933).

${ }^{6}$ Meitner and Philipp, Naturwiss. 21, 286 (1933).

${ }^{7}$ Curie and Joliot, Comptes Rendus 196, 1105 (1933). 


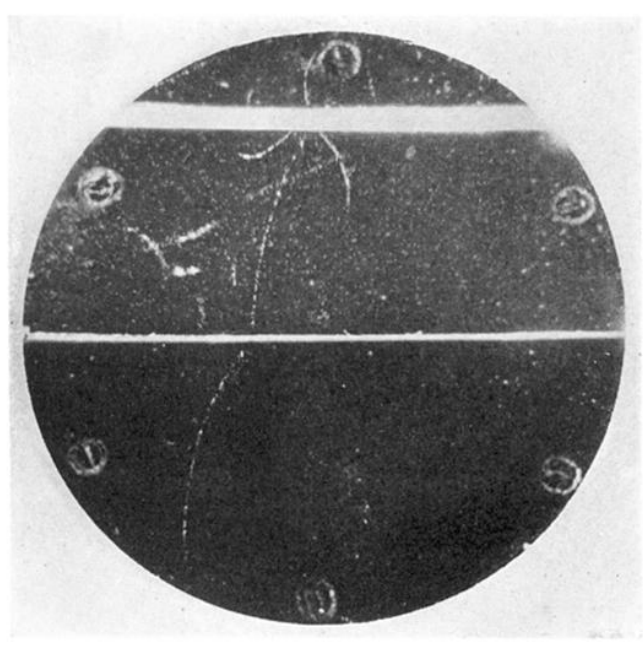

FIG. 1. A positron ejected from a lead plate by gammarays and passing through a $0.5 \mathrm{~mm}$ aluminum plate, showing a loss in energy. On the assumption that its mass is the same as that of a free electron the energy before penetrating the aluminum plate is 820,000 volts and afterward 520,000 volts. 